\title{
Magnetic-field-induced Luttinger liquid
}

\author{
C. Biagini ${ }^{1,2}$, D. L. Maslov ${ }^{2}$, M. Yu. Reizer ${ }^{2}$, and L. I. Glazman ${ }^{3}$ \\ 1) Istituto Nazionale di Fisica della Materia, L.go E. Fermi 2, 50125, Firenze, Italy \\ ${ }^{2)}$ Department of Physics, University of Florida, P. O. Box 118440, Gainesville, Florida 32611-8440 \\ 3) University of Minnesota, Theoretical Physics Institute, 116 Church Street, SE Minneapolis, MN 55455
}

(October 22, 2018)

\begin{abstract}
It is shown that a strong magnetic field applied to a bulk metal induces a Luttinger-liquid phase. This phase is characterized by the zero-bias anomaly in tunneling: the tunneling conductance scales as a power-law of voltage or temperature. The tunneling exponent increases with the magnetic field as $B \ln B$. The zero-bias anomaly is most pronounced for tunneling with the field applied perpendicular to the plane of the tunneling junction.
\end{abstract}

PACS numbers: 71.10Pm,72.15Gd,72.15

A strong magnetic field applied to a bulk metal tends to reduce the effective dimensionality of charge carriers from $3 \mathrm{D}$ to $1 \mathrm{D}$. This feature is most pronounced in the ultra-quantum limit, when only the lowest Landau level remains populated. The reduction of the effective dimensionality in a system of interacting particles is expected to result in a number of unusual phases, which are onedimensional in nature, such as spin-and charge-density waves $(\mathrm{CDW}) \mathrm{e}$ 目, Wigner crystall, excitonic insulator

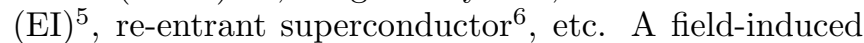
CDW is believed to have been observed in graphite (see, e.g., Ref. [ 7] for an extensive bibliography and discussion). There have been also earlier repgrts of tentative field-induced EI transitions in $\mathrm{Bi}_{1-x} \mathrm{Sb}_{x}$ and $\mathrm{Bi}$.

In this paper, we focus on another field-induced state, which is not related to any instability, but evolves adiabatically from the conventional three-dimensional (3D) Fermi-liquid as the magnetic field increases. This state is a Luttinger Liquid (LL), whose existence can be anticipated from the following simplified picture. In a strong magnetic field, electron trajectories are helices spiraling around the field lines. A bundle of such trajectories with a common center of orbit can be viewed as a $1 \mathrm{D}$ conductor ("wire") with the Fermi velocity $v_{F}^{B}$ determined from the condition of the fixed carrier density $n$ in the bulk,

$$
v_{F}^{B}=2 \pi^{2} n \ell_{B}^{2} / m_{\|} .
$$

Here $\ell_{B}=(e B)^{-1 / 2}$ is the magnetic length, and $m_{\|}$is the effective mass along the field; we assume also full spin polarization of electrons, and use the units with $\hbar=c=1$. In the presence of electron-electron interactions, each "wire", considered separately, is in the LL-state. Interactions with small-momentum transfers among electrons on different "wires" do not change the LL-nature of a single-wire state 10 .

The quantity of primary interest of this paper is the experimentally measurable density of states (DOS) at the sample boundary. We calculate this quantity first by treating perturbatively the interaction between electrons moving in 3D space in the presence of a quantizing magnetic field. We demonstrate that the boundary DOS ex- hibits a characteristic for a Luttinger liquid 19 power-law anomaly at the Fermi level:

$$
\nu(\varepsilon) \propto\left|\varepsilon-\varepsilon_{F}^{B}\right|^{\alpha_{s}},
$$

where $\varepsilon_{F}^{B}=m_{\|}\left(v_{F}^{B}\right)^{2} / 2$ is the Fermi energy of the $1 \mathrm{D}$ motion along the magnetic field.

We establish the correspondence between a 3D electron plasma in a quantizing magnetic field and a Luttinger liquid by considering the electron-electron interaction in the basis of coherent states (CS) on the von Neumann lattice 12 . In this basis, a system of 3D electrons in the ultra-quantum limit is equivalent to a lattice of $1 \mathrm{D}$ wires pointing in the direction of the field and separated by distance $\sqrt{2 \pi}{ }_{B}$, similar to the simplified picture mentioned above 4 . The problem of an array of wires coupled via the long-range Cqulomb interaction [DzyaloshinskiiLarkin (DL) model10] can be solved exactly either via summation of diagram series 10 or bosonization 15 . Utilizing the latter technique, we derive the "bulk" tunneling density of states. This quantity also exhibits a power-law anomaly (2) with a different exponent $\alpha$. Different values of bulk and boundary exponents is another characteristic feature of a LL11. We calculate explicitly the values of the exponents $\alpha$ and $\alpha_{s}$ in the limit of a long-range interaction potential,

$$
\kappa^{B} \ell_{B} \ll 1,
$$

where $\kappa^{B}=\omega_{p} / v_{F}^{B}$ is the (field-dependent) inverse screening length, and $\omega_{p}$ is the zero-field plasma frequency. Condition (3) is satisfied in magnetic fields $B \lesssim B_{0} \equiv \pi^{3} \epsilon n / m_{\|} e^{3}$, where $\epsilon$ is the background dielectric constant. The introduced here characteristic magnetic field $B_{0}$ can be expressed as $B_{0} \simeq B_{q} / r_{s}$ in terms of the magnetic field $B_{q}$ de-populating all but the lowest Landau level, and of the gas parameter $r_{s}$. Under condition (3), we find

$$
\alpha_{s}=2 \alpha \ln \frac{1}{\sqrt{\alpha}}, \quad \alpha=\frac{e^{2}}{2 \pi \epsilon v_{F}^{B}} \equiv \frac{B}{4 B_{0}} .
$$

The tunneling current-voltage characteristic is thus a power-law 


$$
I \propto|V|^{\alpha_{s}+1} \operatorname{sgnV}
$$

for $e|V| \ll \varepsilon_{F}^{B}$. Eq. (1) refers to the situation when the magnetic field is perpendicular to the plane of the tunneling contact. If the field is parallel to the contact plane, electrons move along skipping orbits. Interactions between electrons moving in the same direction do not result in anomalous scaling, therefore there is no tunneling anomaly for this field orientation. This means that by tilting the field one can vary the tunneling exponent from its maximum value, given by Eq. (4), to zero.

Another indication of the tunneling anomaly comes from the gapless behavior of a plasmon in a strong magnetic field. For $\omega_{c} \gg \omega$, where $\omega_{c}=e B / m_{c}$ is the cyclotron frequency, the dispersion relation for a classical magnetoplasmon is given by $\omega^{2}=\left(k_{z}^{2} / k^{2}\right) \omega_{p}^{2}$ [16]. As we see, $\omega \rightarrow 0$ for $k_{z} \rightarrow 0$ at finite $k$. A gapless charge mode slows down the relaxation of an excessive charge added to the conductor in a tunneling event. This mechanism is responsible for the zero-bias anomalies in tunneling into disordered metals and 1D Luttinger liquids. A metal in a strong magnetic field provides one more example of such a behavior.

The LL is destroyed either by backscattering between electrons on different "wires", which results in a CDWinstability, or by formation of bound electron-hole pairs (for the case of a semimetal), which leads to an EIinstability. Also, impurity scattering transfers electrons between the "wires" and thus destroys the 1D motion. The relevant energy scales for these processes is the energy gap, $\Delta$, of either CDW- or EI-origin, and the level broadening, $\Gamma$, respectively. On the other hand, a LLbehavior sets in at energies smaller than the Fermi energy $\varepsilon_{F}^{B}$. The gap $\Delta$ increases with the field, whereas $\varepsilon_{F}^{B}$ decreases with the field. The LL-state should thus exist in the energy interval which narrows down as $B$ increases:

$$
\max \{\Delta(\mathrm{B}), \Gamma\} \lesssim \varepsilon \ll \varepsilon_{\mathrm{F}}^{\mathrm{B}} \propto \mathrm{B}^{-2} .
$$

Before giving the derivation of the results announced above, we would like to discuss their relevance to the experiment. The search for LL-like tunneling anomalies in truly $1 \mathrm{D}$ systems (edges of $2 \mathrm{D}$ electron gas in the EQHE regime 17 , carbon nanotubes 18 , and quantum wires 19 ) has been a very active but remarkably difficult area over the last few years. Compared to experiments on tunneling into $1 \mathrm{D}$ systems, tunneling into a 3D system in the ultraquantum limit has the obvious advantage of the macroscopic system size. It is also advantageous that the tunneling exponent is a function of the external parameters (magnitude and direction of the magnetic field), which can be varied over a wide range.

The choice of the right material is crucial. Among the low-density semimetals (Bi and its alloys and graphite) used conventionally for high-field studies, graphite seems to be the optimal candidate. For $B$ along the c-axis, the ultra-quantum limit is achiered at $B \simeq 7 \mathrm{~T}$. With material parameters for graphite $20\left(m_{\|}=10 m_{0}, \epsilon=6\right.$, and $n=3 \times 10^{18} \mathrm{~cm}^{-3}$ ), Eq. (里) gives $\alpha=0.8$ already at $7 \mathrm{~T}$. For comparison, an anomalously large value of the background dielectric constant $(\epsilon \simeq 100)$ for $\mathrm{Bi} 21$ and a smaller value of $m_{\|}$( $=0.5 m_{0}$ for holes) makes $\alpha$ to be very small: even for $B=100 \mathrm{~T}$, one has $\alpha=0.05$. According to a recent experiment 22 , the field-induced CDW gap in graphite $\Delta \lesssim 1 \mathrm{~K}$ for $B \lesssim 30 \mathrm{~T}$ and takes its maximum value $(\Delta \simeq 10 \mathrm{~K})$ at $B \simeq 50 \mathrm{~T}$. For fields in the range $7 \mathrm{~T} \lesssim B \lesssim 30 \mathrm{~T}$, inequality (6) is satisfied for more than two decades of energies (voltages).

In the limit of weak electron-electron interactions, one can derive the field-induced zero-bias tunneling anomaly (5) by calculating interaction corrections to the transmission coefficient through a tunneling barrier 23. These corrections can be then summed up by using the renormalization group procedure.

Let a potential barrier of transmission coefficient $t_{0} \ll 1$ separate two metallic half-spaces, $z<0$ and $z>0$. The magnetic field is perpendicular to the contact plane. We assume that the base-electrode $(z<0)$ is made of a highdensity metal and therefore is not affected by the magnetic field. Electron-electron interactions are then not important in this half-space and we treat it as a Fermi gas. The counter-electrode $(z>0)$ is a low-density metal in the ultra-quantum magnetic limit. In this half-space, electrons interact via potential $U\left(\mathbf{r}-\mathbf{r}^{\prime}\right)$. Keeping only the leading terms in $t_{0} \ll 1$, the free wave-function in the Landau gauge $\mathbf{A}=(-y B, 0,0)$ for $z>0$ and far away from the barrier is given by

$$
\begin{aligned}
\Psi_{p_{x},-p_{z}}^{0} & =-\left(2 i \sin p_{z} z / \sqrt{L_{z}}\right) \Phi_{p_{x}}^{0}\left(\mathbf{r}_{\perp}\right) \\
\Psi_{p_{x}, p_{z}}^{0} & =t_{0}\left(e^{i p_{z} z} / \sqrt{L_{z}}\right) \Phi_{p_{x}}^{0}\left(\mathbf{r}_{\perp}\right),
\end{aligned}
$$

where $p_{z}=\sqrt{2 m \varepsilon}>0, \mathbf{r}_{\perp} \equiv(x, y)$ and

$\Phi_{p_{x}}^{0}\left(\mathbf{r}_{\perp}\right)=\left(\sqrt{\pi} \ell_{B} L_{x}\right)^{-1 / 2} e^{i p_{x} x} \exp \left[-\left(y+p_{x} \ell_{B}^{2}\right)^{2} / 2 \ell_{B}^{2}\right]$.

The leading correction to the transmission coefficient comes from the exchange interaction 23 , if the interaction potential is smooth on the scale of Fermi wavelength, see Eq. (3).

The correction to the wave caused by the exchange potential $V_{x}$ has the form

$$
\begin{aligned}
\Psi_{p_{x}, p_{z}}(\mathbf{r})= & \Psi_{p_{x}, p_{z}}^{0}(\mathbf{r})+\iint d \mathbf{r}^{\prime} d \mathbf{r}^{\prime \prime} G_{\varepsilon_{z}}^{>}\left(\mathbf{r}, \mathbf{r}^{\prime}\right) \\
& \times V_{x}\left(\mathbf{r}^{\prime}, \mathbf{r}^{\prime \prime}\right) \Psi_{p_{x}, p_{z}}^{0}\left(\mathbf{r}^{\prime \prime}\right),
\end{aligned}
$$

where $G_{\varepsilon_{z}}^{>}\left(\mathbf{r}, \mathbf{r}^{\prime}\right)$ is the Green's function of the free Schrödinger equation in the half-space $z>0$, and $\varepsilon_{z} \equiv$ $p_{z} / 2 m^{2}$ is the energy of electron motion along the field. The integration in (8) goes only over those regions where electrons interact, i.e., $z^{\prime}, z^{\prime \prime} \geq 0$.

The renormalized transmission coefficient is extracted from the asymptotic form of $\Psi_{p_{x}, p_{z}}$ for $z \rightarrow+\infty$ which, in its turn, is determined by the asymptotic behavior of $G_{\varepsilon_{z}}^{>}\left(\mathbf{r}, \mathbf{r}^{\prime}\right)$ [see Eq. (8)]. The asymptotic form of 
the Green's function in the infinite space can be written as $\left.G_{p_{z}}\left(\mathbf{r}, \mathbf{r}^{\prime}\right)\right|_{z \rightarrow+\infty}=G_{\|}\left(z, z^{\prime}, p_{z}\right) G_{\perp}\left(\mathbf{r}_{\perp}, \mathbf{r}_{\perp}^{\prime}\right)$, where $G_{\varepsilon_{z}}^{\| \mid}\left(z, z^{\prime}\right)=\left(m_{||} / i p_{z}\right) e^{i p_{z}\left|z-z^{\prime}\right|}$ is the Green's function for the $1 \mathrm{D}$ motion along the $z$-axis, and

$$
G_{\perp}\left(\mathbf{r}_{\perp}, \mathbf{r}_{\perp}^{\prime}\right)=\frac{1}{2 \pi \ell_{B}^{2}} e^{-\left[\mathbf{r}_{\perp}-\mathbf{r}_{\perp}^{\prime}\right]^{2} / 4 \ell_{B}^{2}} e^{-i\left(y+y^{\prime}\right)\left(x-x^{\prime}\right) / 2 \ell_{B}^{2}} .
$$

The asymptotic form of $G_{\varepsilon_{z}}^{>}\left(\mathbf{r}, \mathbf{r}^{\prime}\right)$ is then obtained via the method of images

$\left.G_{\varepsilon_{z}}^{>}\left(\mathbf{r}, \mathbf{r}^{\prime}\right)\right|_{z \rightarrow+\infty}=\left[G_{p_{z}}^{\|}\left(z, z^{\prime}\right)-G_{p_{z}}^{\|}\left(z,-z^{\prime}\right)\right] G_{\perp}\left(\mathbf{r}_{\perp}, \mathbf{r}_{\perp}^{\prime}\right)$.

The exchange potential is given by

$$
\begin{aligned}
V_{x}\left(\mathbf{r}, \mathbf{r}^{\prime}\right)= & -U\left(\mathbf{r}-\mathbf{r}^{\prime}\right) \int_{0}^{p_{F}^{B}} \frac{d p_{z}}{2 \pi} \int_{-\infty}^{\infty} \frac{d p_{x}}{2 \pi}\left[\Psi_{p_{x},-p_{z}}^{0}\left(\mathbf{r}^{\prime}\right)\right]^{*} \\
& \times \Psi_{p_{x},-p_{z}}^{0}(\mathbf{r}) \\
& \approx-U\left(\mathbf{r}-\mathbf{r}^{\prime}\right) \frac{\sin p_{F}^{B}\left(z+z^{\prime}\right)}{\pi\left(z+z^{\prime}\right)} G_{\perp}\left(\mathbf{r}_{\perp}, \mathbf{r}_{\perp}^{\prime}\right), \quad(10)
\end{aligned}
$$

where $p_{F}^{B}=m_{\|} v_{F}^{B}$. In Eq. (10), we retained only that term which at $p_{z} \approx p_{F}^{B}$ leads to a logarithmic divergence in the integral over $z+z^{\prime}$ in Eq. (8).

The transmission coefficient in the first order with respect to interactions is

$$
t=t_{0}\left(1-\frac{e^{2}}{2 \pi \epsilon v_{F}^{B}} \ln \frac{1}{\kappa^{B} \ell_{B}} \ln \frac{1}{\ell_{B}\left|p_{z}-p_{F}^{B}\right|}\right),
$$

where the inverse screening radius

$$
\kappa^{B}=\omega_{p} / v_{F}^{B}=2 \alpha \ell_{B}^{-1} \propto B .
$$

This result is valid if both logarithmic factors are large. The contribution $t$ from the Hartree term is smaller than (11) by $\ln \left(1 / \kappa^{B} \ell_{B}\right)$. The higher order terms in the expansion of $t$ can he summed up via the renormalization group procedure23. The result is

$$
t \propto\left|p_{z}-p_{F}^{B}\right|^{\alpha_{s} / 2} \propto\left|\varepsilon-\varepsilon_{F}^{B}\right|^{\alpha_{s} / 2} .
$$

As $I \propto|t|^{2} V$, Eq. (13) yields Eq. (5) with exponent (4). The perturbative result (13) is valid for $\alpha \ll 1$, which means that $\alpha_{s}$ is also small within this approach.

We now demonstrate how the LL-state emerges in the coherent state formulation. The method of bosonization, applied in this formulation, provides a simple tool for calculation the "bulk" 1D density of states11. Also, it allows us to estimate the tunneling exponents for stronger interaction $(\alpha \gtrsim 1)$. The $\Psi$-operator is expanded over the coherent states basis

$$
\Psi(\mathbf{r})=\sum_{N} \sum_{\mathbf{R}} a_{N \mathbf{R}}^{\dagger}(z) \chi_{N \mathbf{R}}\left(\mathbf{r}_{\perp}\right),
$$

where $\mathbf{r}=\left(z, \mathbf{r}_{\perp}\right), \mathbf{R}=\left(R_{x}, R_{y}\right)$. A coherent state $\chi_{N \mathbf{R}}\left(\mathbf{r}_{\perp}\right)$ is a simultaneous eigenstate of the energy operator

$$
\left(2 m_{c}\right)^{-1}\left(\mathbf{p}_{\perp}-e \mathbf{A}\right)^{2} \chi_{N \mathbf{R}}=\omega_{c}(N+1 / 2) \chi_{N \mathbf{R}},
$$

and of the "guiding center" operator

$$
\left(\hat{x}_{0}-i \hat{y}_{0}\right) \chi_{N \mathbf{R}}=\left(R_{x}-i R_{y}\right) \chi_{N \mathbf{R}},
$$

where $\hat{x}_{0}=\hat{x}+\left(m \omega_{c}\right)^{-1}\left(\hat{p}_{y}-e A_{y}\right)$ and $\hat{y}_{0}=\hat{y}-$ $\left(m \omega_{c}\right)^{-1}\left(\hat{p}_{x}-e A_{x}\right)$ are the operators corresponding to the classical coordinates of guiding center 24 . In the symmetric gauge, $\mathbf{A}=(1 / 2) \mathbf{B} \times \mathbf{r}$ an explicit form of the CS corresponding to $N=0$ is 24

$$
\chi_{0 \mathbf{R}}\left(\mathbf{r}_{\perp}\right)=\frac{1}{\sqrt{2 \pi} \ell_{B}} \exp \left[-\frac{\left(\mathbf{r}_{\perp}-\mathbf{R}\right)^{2}+2 i \mathbf{r}_{\perp} \wedge \mathbf{R}}{4 \ell_{B}^{2}}\right],
$$

where $\mathbf{a} \wedge \mathbf{b}=a_{x} b_{y}-a_{y} b_{x}$.

As any set of coherent states, the set of $\chi_{N \mathbf{R}}$ remains (over) complete even if defined on a subset of points in the complex plane, $R_{x}+i R_{y}$, rather than on the whole plane. The von Neumann theorem 25 allows one to choose this subset as sites of a $2 \mathrm{D}$ square lattice. To fix the value of the lattice spacing, one notices that a total of $n$ particles per unit volume are now distributed over "wires", which are parallel to the magnetic field and cross the plane perpendicular to the field at the sites of the von Neumann lattice. For $N=0$, the number density per unit length of each "wire" is $p_{F}^{B} / \pi$, their the areal density is $1 / s^{2}$, thus $n=p_{F}^{B} / \pi s^{2}$. Comparing this equation to (1), one gets $s=\sqrt{2 \pi} \ell_{B}$. This is a maximum value of the lattice spacing for which the set is still complete. States of the CS basis on the von Neumann lattice describe particles which are localized on the lattice sites in the direction transverse to the field but free to move along the field. This picture is in a close resemblance to the classical trajectories spiraling around the field lines.

Coherent states are not orthogonal. In particular,

$$
\left\langle\chi_{0 \mathbf{R}} \mid \chi_{0 \mathbf{R}^{\prime}}\right\rangle=\frac{1}{2} \exp \left[-\frac{\left(\mathbf{R}-\mathbf{R}^{\prime}\right)^{2}+2 i \mathbf{R} \wedge \mathbf{R}^{\prime}}{4 \ell_{B}^{2}}\right],
$$

which can be replaced by $2 \pi \ell_{B}^{2} \delta^{2}\left(\mathbf{R}-\mathbf{R}^{\prime}\right)$ in the limit $\ell_{B} \rightarrow 0$. Only in this limit the coherent set diagonalizes the quadratic part of the Hamiltonian. Physically, this limit means that $\ell_{B}$ is much less than the characteristic value of $\left|\mathbf{R}-\mathbf{R}^{\prime}\right|$. For interacting electrons, typical $\left|\mathbf{R}-\mathbf{R}^{\prime}\right|$ is of the order of the screening radius $1 / \kappa^{B}$. Thus the approach based on coherent states is valid for a long-range interaction as defined by condition (3).

Using asymptotic orthogonality of the coherent states, one arrives at the effective 1D Hamiltonian (from now on we concentrate on $N=0$ and suppress index $N$ )

$$
\begin{aligned}
H & =\sum_{\mathbf{R}} \int d z a_{\mathbf{R}}^{\dagger}(z)\left[\frac{\omega_{c}}{2}+\frac{p_{z}^{2}}{2 m_{\| l}}\right] a_{\mathbf{R}}(z) \\
& +\frac{e^{2}}{2 \epsilon} \sum_{\mathbf{R}, \mathbf{R}^{\prime}} \int d z d z^{\prime} \frac{a_{\mathbf{R}}^{\dagger}(z) a_{\mathbf{R}^{\prime}}^{\dagger}\left(z^{\prime}\right) \hat{a}_{\mathbf{R}^{\prime \prime}}\left(z^{\prime}\right) a_{\mathbf{R}}(z)}{\sqrt{\left(\mathbf{R}-\mathbf{R}^{\prime}\right)^{2}+\left(z-z^{\prime}\right)^{2}}} .
\end{aligned}
$$


Due to the long-range nature of the Coulomb potential, it suffices to keep only the forward scattering processes in (16) (both for $\mathbf{R}=\mathbf{R}^{\prime}$ and $\mathbf{R} \neq \mathbf{R}^{\prime}$ ), in which case (16) is identical to the DL modello Backscattering for $\mathbf{R} \neq \mathbf{R}^{\prime}$ leads to a CDW instability 13 . The forward scattering part of (16) is diagonalized via bosonizing the fermions

$$
a_{\mathbf{R}}=: e^{-i\left(p_{F}^{B} z+\sqrt{\pi}\left\{\phi \mathbf{R}+\theta_{\mathbf{R}}\right\}\right)}+e^{i\left(p_{F}^{B} z+\sqrt{\pi}\left\{\phi_{\mathbf{R}}-\theta_{\mathbf{R}}\right\}\right)}:,
$$

where $\left[\phi_{\mathbf{R}}\left(z_{1}\right), \partial_{z_{2}} \theta_{\mathbf{R}^{\prime}}\left(z_{2}\right)\right]=i \delta_{\mathbf{R}, \mathbf{R}^{\prime}} \delta\left(z_{1}-z_{2}\right)$. The result of a straightforward calculation for the equal-point (Matsubara) Green's function is

$$
\begin{aligned}
\mathcal{G}(\tau) & \sim \tau^{-1} e^{-f(\tau)}, \\
f(\tau) & =2 \pi^{2} \ell_{B}^{2} \int_{\mathrm{BZ}} \frac{d^{2} q_{\perp}}{(2 \pi)^{2}} \int_{0}^{\Lambda} \frac{d q_{z}}{2 \pi} \frac{(u-1)^{2}}{u} \frac{1-e^{-u v_{F}^{B} q_{z} \tau}}{q_{z}}, \\
u^{2} & \equiv 1+\left(\kappa^{B}\right)^{2} \sum_{\mathbf{G}}(\mathbf{Q}+\mathbf{G})^{-2}, \mathbf{Q}=\left(\mathbf{q}_{\perp}, q_{z}\right) .
\end{aligned}
$$

Here the $q_{\perp}$-integration goes over the Brillouin zone (BZ) of the von Neumann lattice, $\mathbf{G}$ are the reciprocal lattice vectors, $\Lambda$ is the cutoff, and $u v_{F}^{B}$ has the meaning of a plasmon velocity. Typical $q_{z}$ in $f(\tau)$ are determined by the low-energy scale of the problem $(\max \{\mathrm{T}, \mathrm{eV}\})$ and thus small, which allows one to neglect $q_{z}$ in function $u$. The DOS reduces to the form (2) with an exponent

$$
\alpha=\pi \ell_{B}^{2} \int_{\mathrm{BZ}} \frac{d^{2} q_{\perp}}{(2 \pi)^{2}} \frac{(u-1)^{2}}{u} .
$$

For $\kappa^{B} \ell_{B} \ll 1$, the sum over the reciprocal vectors in (18) is dominated by the $\mathbf{G}=0$ term so that $u^{2} \approx 1+\left(\kappa^{B} / q_{\perp}\right)^{2}$. The main contribution to the integral over $q_{\perp}$ in (18) comes from the region $q_{\perp} \simeq \kappa^{B} \ll 1 / \ell_{B} \simeq G$, thus the integration can be extended over the entire $q$-space. The bulk DOS behaves as $\left|\varepsilon-\varepsilon_{F}^{B}\right|^{\alpha}$, where $\alpha$ is given in Eq. (4).

Note that in the main contribution to $\alpha$ [see Eq. (18)], a typical value of $u-1$ is of the order of unity. This indicates a non-perturbative nature of the result for $\alpha$. The lowest-order perturbation theory, applied to the bulk case, would have given $\alpha \propto e^{4}$, whereas the correct result is $\alpha \propto e^{2}$.

Tunneling into the end of a semi-infinite sample can be treated by imposing the boundary condition on the current operator: $j_{\mathbf{R}}=(1 / i \sqrt{\pi}) \partial_{\tau} \phi_{\mathbf{R}}=(1 / \sqrt{\pi}) \partial_{x} \theta_{\mathbf{R}}=0$ for $z=0$. This translates into the boundary conditions for the propagators: $\mathcal{P}_{\mathbf{R}}^{\rho}\left(z, z^{\prime}, \tau\right)=\left\langle\rho_{\mathbf{R}}(z, \tau) \rho_{\mathbf{R}}\left(z^{\prime}, 0\right)-\right.$ $\left.\rho_{\mathbf{R}}^{2}(z, 0)\right\rangle$, where $\rho=\phi, \theta$. $\mathcal{P}_{\mathbf{R}}^{\rho}$ can be constructed from the propagators for the infinite medium, $P_{\mathbf{R}}^{\rho}$, by using the method of images. Evidently, $\mathcal{P}_{\mathbf{R}}^{\phi}=0$ at the boundary whereas $\mathcal{P}_{\mathbf{R}}^{\theta}(0,0, \tau)=\pi^{-1} \int d q_{z} P_{\mathbf{R}}^{\theta}\left(q_{z}, \tau\right)$. The DOS at the boundary is again of form (2) but with an exponent

$$
\alpha_{s}=2 \pi \ell_{B}^{2} \int_{\mathrm{BZ}} \frac{d^{2} q_{\perp}}{(2 \pi)^{2}}(u-1) .
$$

In contrast to the bulk case, the logarithmically divergent integral over $q_{\perp}$ in (19) is cut off at the BZ boundary, i.e., for $q_{\perp} \simeq 1 / \ell_{B}$. Therefore, the condition of asymptotic orthogonality of CS is satisfied only with the logarithmic accuracy. The result for $\alpha_{s}$ is given by Eq. (仼).

In the strong-coupling case $(\alpha \gtrsim 1)$, typical $q_{\perp} \simeq 1 / \ell_{B}$, which means that the approach based on CS becomes invalid quantitatively. However, one can still estimate tunneling exponents (both for the "bulk" and "edge tunneling) as $\tilde{\alpha}=C \sqrt{e^{2} / v_{F}^{B}}$, where the numerical constant $C \simeq 1$ cannot be determined by this method.

We are grateful to I. L. Aleiner, A. I. Larkin, and Z. Tešanović for interesting discussions. C. B. acknowledges the partial support of the project COFIN98-MURST. The work at the University of Florida was supported by the NHMFL In-House Research Program, NSF (DMR970338) and Research Corporation (RI0082). L. I. G. acknowledges the support from NSF DMR-9731756 and DMR-9812340.

* temporary address.

${ }^{1}$ V. Celli and N. D. Mermin, Phys. Rev. 140, A839 (1965).

${ }^{2}$ H. Yoshioka and H. Fukuyama, J. Phys. Soc. of Jpn. 50, 725 (1981).

${ }^{3}$ V. M. Yakovenko Phys. Rev. B 47, 8851 (1993).

${ }^{4}$ J. I. Kaplan and M. L. Glasser, Phys. Rev. Lett. 28, 1077 (1972); W. G. Kleppmann and R. J. Elliott, J. Phys. C 8, 2729 (1975).

${ }^{5}$ A. A. Abrikosov, J. Low Temp. Phys. 2, 37 (1970); ibid., 175 (1970).

${ }^{6}$ M. Rasolt and Z. Tešanović, Rev. Mod. Phys. 64, 709 (1992).

${ }^{7}$ Y. Shimamoto, N. Miura and H. Nojiri, J. of Phys.: Condensed Matter 10, 11289 (1998).

${ }^{8}$ N. B. Brandt and S. M. Chudinov, Pi'sma Zh. Èksp. Teor. Fiz. 13, 146 (1971) [JETP Lett. 13, 102 (1971)].

${ }^{9}$ S. Mase and T. Sakai, J. Phys. Soc. Jpn. 31, 730 (1971).

${ }^{10}$ I. E. Dzyaloshinskii and A. I. Larkin, Zh. Èksp. Teor. Fiz. 65, 411 (1973) [Sov. Phys. JETP 38, 202 (1974)].

${ }^{11}$ C. L. Kane and M. P. Fisher, Phys. Rev. Lett. 76, 3192 (1996).

12 The Luttinger liquid behavior for a 3D electron plasma subject to a quantizing field was conjectured in Ref. [13].

${ }^{13}$ V. S. Babichenko and A. N. Kozlov, Pi'sma Zh. Èksp. Teor. Fiz. 61, 722 (1995) [JETP Lett. 61, 737 (1995)].

${ }^{14}$ In contrast to earlier work $\$$, in which a Wigner transition was viewed as actual breaking of the electron liquid into "charged rods", the "wires" emerging in the CS description are fictitious. This representation is merely an illustration which emphasizes small spatial overlap of states with guiding centers separated by a distance exceeding $\ell_{B}$.

${ }^{15}$ H. J. Schulz, J. Phys. C 15, 6769 (1983).

${ }^{16}$ E. M. Lifshits and L. P. Pitaevskii, Physical kinetics (Pergamon Press, Oxford, New York, 1981).

17 A. Chang, L. N. Pfeiffer, and K. W. West, Phys. Rev. Lett. 77, 2538 (1996). 
18 S. J. Tans et al., Nature 394, 761 (1998); M. Bockrath et al., ibid. 397, 598 (1999).

19 O. M. Auslender et al., Phys. Rev. Lett. 84, 1764 (2000).

${ }^{20}$ N. B. Brandt, S. M. Chudinov and Ya. G. Pogorelov Semimetals 1. Graphite and Its Compounds, (NorthHolland, Amsterdam, 1988).

${ }^{21}$ V. S. Edel'mann, Usp. Fiz. Nauk. 123, 257 (1977) [Sov. Phys. Usp. 20, 819 (1977)].
${ }^{22}$ H. Yaguchi and J. Singleton, Phys. Rev. Lett. 81, 5193 (1998).

23 Dongxiao Yue, L. I. Glazman and K. A. Matveev, Phys. Rev. B B49, 1996 (1994).

${ }^{24}$ I. A. Malkin and V. I. Man'ko, Zh. Èksp. Teor. Fiz. 55, 1014 (1968) [ Sov. Phys. JETP 28, 527 (1969)].

${ }^{25}$ See, e.g., A. M. Perelomov, Generalized Coherent States and Their Applications, (Springer, 1986), Ch. 1.4. 